\title{
Effects of Exercise on Acute Kidney Injury Biomarkers and the Potential Influence of Fluid Intake
}

\author{
Loris Allan Juett Lewis J. James Stephen Andrew Mears \\ School of Sport, Exercise and Health Sciences, Loughborough University, Loughborough, UK
}

\section{Keywords}

Acute kidney injury - Chronic kidney disease · Exercise · Neutrophil gelatinase-associated lipocalin · Kidney injury molecule- $1 \cdot$ Hydration · Fructose

\begin{abstract}
Acute kidney injury (AKI) incidence (diagnosed by changes in serum creatinine [Cr]) following prolonged endurance events has been reported to be anywhere from 4 to $85 \%$, and hypohydration may contribute to this. Whilst an increase in serum $\mathrm{Cr}$ indicates impaired kidney function, this might be influenced by muscle damage. Therefore, the use of other AKI biomarkers which can detect renal tubular injury may be more appropriate. The long-term consequences of AKI are not well understood, but there are some potential concerns of an increased subsequent risk of chronic kidney disease (CKD). Therefore, this brief review explores the effects of exercise training/competition on novel AKI biomarkers and the potential influence of fluid intake. The increase in novel AKI biomarkers following prolonged endurance events suggests renal tubular injury. This is likely due to the long duration and relatively high exercise intensity, producing increased sympathetic tone, body temperature, hypohydration, and muscle damage. Whilst muscle damage appears to be an important factor in the pathophysiology of exercise-associated $\mathrm{AKI}$, it may require coexisting hypohydration. Fluid intake
\end{abstract}

karger@karger.com www.karger.com/anm

Karger $\frac{1}{\%}$
(C) 2021 The Author(s)

Published by S. Karger AG, Basel

This article is licensed under the Creative Commons AttributionNonCommercial-NoDerivatives 4.0 International License (CC BYNC-ND) (http://www.karger.com/Services/OpenAccessLicense) Usage and distribution for commercial purposes as well as any distribution of modified material requires written permission. seems to play a role in exercise-associated AKI, as maintaining euhydration with water ingestion during simulated physical work in the heat appears to attenuate rises in AKI biomarkers. The composition of fluid intake may also be important, as high-fructose drinks have been shown to exacerbate AKI biomarkers. However, it is yet to be seen if these findings are applicable to athletes performing strenuous exercise in a temperate environment. Additionally, further work should examine the effects of repeated bouts of strenuous exercise on novel AKI biomarkers.

(C) 2021 The Author(s)

Published by S. Karger AG, Basel

\section{Introduction}

Following prolonged endurance events, the incidence of acute kidney injury (AKI), defined as a rapid decline in kidney function, has been reported to be anywhere from 4 to $85 \%$ [1-5]. This variable, but often high, incidence is likely due to a combination of factors, including muscle damage, sympathetic tone, body temperature, and hypohydration [1]. Of these factors, hypohydration may be of particular interest, as it is commonly seen during prolonged endurance events [6] and is relatively easy to manipulate (i.e., consume fluid to maintain euhydration). If optimizing hydration status is able to reduce the extent of 
AKI, then this could have implications for long-term renal function, as there are concerns that repeated AKIs (even subclinical AKIs) could increase the subsequent risk of chronic kidney disease (CKD), which describes irreversibly impaired kidney function [7]. This theory has been proposed to explain the epidemic of CKD seen in Central America among sugarcane workers, who are regularly exposed to factors that increase the risk of AKI [8]. This is of concern because CKD is associated with an increased risk of cardiovascular disease and mortality [9]. This topic, specifically kidney injury caused by working in the heat and the subsequent risk of CKD in labourers, was the focus of a recent comprehensive review by Schlader et al. [10], which will provide an excellent background for this brief, invited review. The present review will focus on the impact of exercise training/competition on AKI biomarkers in athletic populations, as well as exploring the influence of fluid intake.

In 2017, Hodgson et al. [11] conducted a systematic review of the literature observing AKI following endurance events, with a focus on serum creatinine $(\mathrm{Cr})$. They reported that increases in serum $\mathrm{Cr}$ concentrations were seen following endurance events, but that the long-term implications were unknown [11]. Serum $\mathrm{Cr}$, the principal biomarker used to clinically diagnose AKI, is a muscle breakdown product filtered by the kidneys and excreted in urine, meaning a rise, in many settings, indicates impaired kidney function. While this impaired kidney function normally indicates renal injury, in the context of prolonged endurance events, the rise in serum $\mathrm{Cr}$ concentrations post-exercise may simply be due to increased muscle breakdown (i.e., increased production) $[1,11]$ and/or a temporary reduction in renal blood flow (RBF) (i.e., reduced clearance) [12]. In this context, the use of other AKI biomarkers may be more appropriate to detect renal tubular injury [13]. Therefore, this brief review will focus on the responses of such biomarkers to exercise training/ competition.

\section{Novel Biomarkers of AKI}

While there are many potential candidate biomarkers of AKI, including neutrophil gelatinase-associated lipocalin (NGAL), kidney injury molecule-1 (KIM-1), interleukin-18, liver-type fatty acid binding protein, insulinlike growth factor binding protein 7 and tissue inhibitor metalloproteinase 2, NGAL and KIM-1 have received the most attention for exercise-associated AKI, as well as having been studied in a variety of clinical settings [13].
NGAL and KIM-1 expression are upregulated following AKI, which is thought to aid the proliferation of tubular cells [13]. An advantage of using NGAL and KIM-1 over serum $\mathrm{Cr}$ is that they may be able to indicate the location of kidney injury, as a rise in urinary KIM-1 (uKIM-1) indicates injury to the proximal tubule [13], whereas a rise in urinary NGAL (uNGAL) indicates injury mainly to the distal nephron [14]. Increases in NGAL and KIM-1 can be detected in blood or urine, but it is thought that the urinary forms may be more specific for determining AKI, as NGAL and KIM-1 are also expressed in organs other than the kidneys [13-15]. Therefore, this brief review will focus on UNGAL and uKIM-1.

An issue for consideration when studying urinary biomarkers is that the concentration of urine itself can vary substantially, and thus a rise in a urinary biomarker may be due to an increase in urine concentration, rather than an increase in biomarker production. To account for this, a variety of corrections have been applied in the literature, including correction for urinary $\mathrm{Cr}$ and urine osmolality $[5,16-21]$. When measured in close proximity to exercise, it is thought that urine osmolality may be the more appropriate correction, as urinary $\mathrm{Cr}$ may be increased due to muscle damage $[17,19]$.

\section{Exercise and RBF}

The previously mentioned risk factors for exercise-associated AKI may all contribute to AKI by reducing RBF $[12,22,23]$. A reduction in $\mathrm{RBF}$ may lead to renal ischaemia and subsequent renal ATP depletion, which can initiate AKI [24]. During exercise, increased sympathetic tone serves to increase blood flow to the skeletal muscles, while increased core body temperature increases blood flow to the skin, both of which may reduce $\operatorname{RBF}[12,22]$. Indeed, this notion is supported by the finding that RBF decreases as exercise intensity increases [12]. Additionally, if exercise involves repeated eccentric muscle contractions, it can cause skeletal muscle damage [25], which may result in extracellular fluid entering the muscle cells [23]. These effects may decrease plasma volume and activate the renin-angiotensin-aldosterone system (RAAS), which may contribute to reduced RBF during exercise [23]. Damage to skeletal muscle may also lead to leakage of cellular contents into the circulation, including myoglobin, which is thought to scavenge nitric oxide, potentially resulting in renal vasoconstriction [23]. Furthermore, hypohydration, although a modifiable factor, is commonly present towards the end of endurance events
Juett/James/Mears 
Fig. 1. Schematic representing how exercise may reduce renal blood flow and thus increase the risk of AKI. AKI, acute kidney injury.

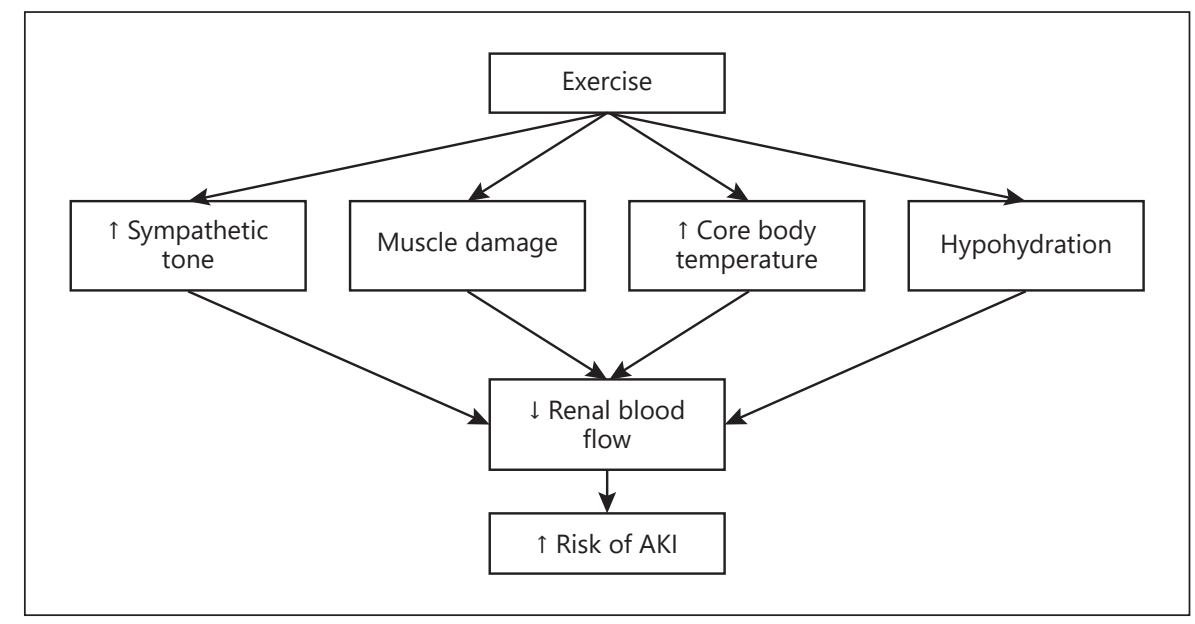

[6]. Typically, hypohydration decreases plasma volume (resulting in subsequent RAAS activation), increases circulating vasopressin, and increases core body temperature during exercise, all of which may further reduce RBF [22, 23, 26-29]. During exercise, these factors may combine to reduce RBF (Fig. 1), which has been reported to fall by as much as $75 \%$ during vigorous exercise [12].

\section{The Effects of Exercise on Novel AKI Biomarkers}

In agreement with the often high incidence of AKI (as defined by serum $\mathrm{Cr}$ ) measured following prolonged endurance events, increases in uNGAL $[3,16,30]$ and uKIM$1[3,30]$ have also been reported after these events. These increases appear to be due to increased biomarker production, rather than simply urine concentration, as in one of these studies, urine specific gravity did not change from pre- to post-marathon [3]. Additionally, in another study, the increase in uNGAL from pre- to post-ultramarathon remained significant after correction for urinary $\mathrm{Cr}$ [16]. Taken together, these findings suggest that prolonged endurance events may cause renal tubular injury, rather than just muscle damage and/or a temporary reduction in RBF.

Given that UNGAL and uKIM-1 concentrations are sometimes only presented in their uncorrected form [3, 30 ], and that when they are corrected, it is often only for urinary $\operatorname{Cr}[5,16,17,21]$, which may be inappropriate post-exercise $[17,19]$, this paragraph will compare uncorrected concentrations between studies. Prolonged endurance events, such as marathons and ultramarathons, appear to be the forms of exercise that produce the highest post-exercise UNGAL concentrations $[3,5,15-21,30$
32]. This may be due to the combination of long duration and relatively high-intensity exercise. This theory could explain the comparatively lower UNGAL concentrations reported after an $800 \mathrm{~m}$ run (high intensity, but very short duration; [17]) and prolonged walking (long duration, but low intensity; [18]). Comparing the response of uKIM- 1 to different forms of exercise is more challenging as there is less data available and there appears to be higher variability in baseline readings $[3,18,19,30]$.

Although exercise duration and intensity appear to be important factors determining the extent of kidney injury, the mechanisms are not fully understood. Increases in exercise duration and intensity are likely to align with increases in sympathetic tone, muscle damage and core body temperature. For example, Bongers et al. [19] documented greater rises in UNGAL and osmolality-corrected uNGAL following $107 \mathrm{~min}$ of exercise, compared to after the initial $30 \mathrm{~min}$ of this exercise, but the lack of a control group meant that no single risk factor could be isolated. Using a crossover design, Junglee et al. [15] demonstrated that UNGAL following exercise in the heat was significantly higher when the exercise was preceded by a bout of downhill muscle-damaging running (compared to flat less-muscle-damaging running). It has also been shown that continuous upper body cooling during exercise in the heat attenuated the increase in UNGAL [32]. The findings from these crossover studies suggest that muscle damage is an important factor in the pathophysiology of exercise-associated AKI and that an increase in core body temperature exacerbates kidney injury. A summary of the studies mentioned in this section of the review that examined the effect of exercise training/competition on uKIM1 and/or uNGAL concentrations is presented in Table 1. 
Table 1. A summary of the studies referenced in the body text that examine the effect of exercise training/competition on uNGAL and/ or uKIM-1 concentrations

\begin{tabular}{|c|c|c|c|c|c|}
\hline Study & $\begin{array}{l}\text { Sample } \\
\text { size }\end{array}$ & Exercise & uNGAL, ng/mL & $\mathrm{uKIM}-1, \mathrm{ng} / \mathrm{mL}$ & $\begin{array}{l}\text { AKI } \\
\text { incidence, } \\
\%\end{array}$ \\
\hline Lippi et al. [16] & 16 & $60 \mathrm{~km}$ ultramarathon $\left(6-8^{\circ} \mathrm{C}\right)$ & $\begin{array}{l}\text { Pre-EX: }[4.4,0.5-33.9] \\
\text { Post-EX: }[35.6,12.5-86.3]\end{array}$ & $\mathrm{nr}$ & 38 \\
\hline Junglee et al. [17] & 20 & $800 \mathrm{~m}$ run & $\begin{array}{l}\text { Pre-EX: } \sim 3 \\
25 \text { minPost-EX: }[12.8,6.2-23.2] \\
2 \text { h post-EX: }[0.8,0.02-3.3]\end{array}$ & $\mathrm{nr}$ & $\mathrm{nr}$ \\
\hline Junglee et al. [15] & 10 & $\begin{array}{l}40 \text { min } \mathrm{HS} \text { run }\left(65 \% \mathrm{VO}_{2} ; 33^{\circ} \mathrm{C}\right) \text { preceded } \\
\text { by either } 60 \text { min run }\left(65 \% \mathrm{VO}_{2} ; 20^{\circ} \mathrm{C}\right) \text { at } \\
-10 \%(\mathrm{MD}) \text { or }+1 \% \text { gradient }(\mathrm{CON}) \\
\text { (crossover) }\end{array}$ & $\begin{array}{l}\text { MD: } \\
\text { Pre-EX: }[\sim 1.9] \\
\text { Post-HS: }[15.0] \\
\text { CON: } \\
\text { Pre-EX: }[\sim 3.0] \\
\text { Post-HS: }[\sim 3.0]\end{array}$ & $\mathrm{nr}$ & $\begin{array}{l}\text { MD: } 50 \\
\text { CON: } 0\end{array}$ \\
\hline Bongers et al. [18] & 60 & $\begin{array}{l}\text { 30, } 40 \text {, or } 50 \mathrm{~km} \text { walks for } 3 \text { consecutive } \\
\text { days }\end{array}$ & $\begin{array}{l}\text { Pre-EX: }[9.2,5.2-14.7] \\
\text { Post day } 1:[20.7,11.0-37.2] \\
\text { Post day } 3:[14.2,8.0-26.3]\end{array}$ & $\begin{array}{l}\text { Pre-EX: }[2.6,1.4-6.0] \\
\text { Post day } 1:[5.2,2.4-9.1] \\
\text { Post day } 3:[2.9,1.4-6.4]\end{array}$ & $\begin{array}{l}\text { Day 1: } 10 \\
\text { Day 3: } 0\end{array}$ \\
\hline Bongers et al. [19] & 35 & $\begin{array}{l}30 \text {-min cycle (acute) }\left(20^{\circ} \mathrm{C}\right) \text { followed by } \\
\text { cycle }\left(25^{\circ} \mathrm{C}\right) 120 \mathrm{~min} \text { or until } 3 \% \text { HYP } \\
\text { (prolonged) }\end{array}$ & $\begin{array}{l}\Delta \text { Pre-EX to post-acute: }[1.0,0.0-8.0] \\
\Delta \text { Pre-EX to post-prolonged: } \\
{[11.1,0.7-22.6]}\end{array}$ & $\begin{array}{l}\Delta \text { Pre-EX to post-acute: } \\
{[0.7,-0.2-2.1]} \\
\Delta \text { Pre-EX to post-prolonged } \\
{[3.0,0.8-7.6]}\end{array}$ & $\mathrm{nr}$ \\
\hline Butts [20] & 18 & $\begin{array}{l}\text { Eccentric knee flexions } \\
(10 \times 10 \text { repetitions) followed by } \\
60 \text {-min run }\left(\sim 60 \% \mathrm{VO}_{2} ; 33^{\circ} \mathrm{C}\right) \text { in } \mathrm{EU} \\
\text { and } \mathrm{HYP} \text { conditions }(\mathrm{crossover})\end{array}$ & $\begin{array}{l}\text { EU: } \\
\text { Pre-EX: }(\sim 5.0) \\
\text { Post-EX: }(\sim 6.0) \\
21.5 \text { h post-EX: }(\sim 7) \\
\text { HYP: } \\
\text { Pre-EX }(\sim 12) \\
\text { Post-EX }(\sim 12) \\
21.5 \text { h post-EX }(\sim 7)\end{array}$ & $\mathrm{nr}$ & $\mathrm{nr}$ \\
\hline Poussel et al. [5] & 24 & 120-km trail race & $\begin{array}{l}\text { Pre-EX: }[20.1] \\
\text { Post-EX: [53.1] }\end{array}$ & $\mathrm{nr}$ & 4 \\
\hline
\end{tabular}

AKI incidence was defined as a 1.5 -fold and/or $0.3 \mathrm{mg} / \mathrm{dL}$ increase in serum Cr from pre-exercise. Data are presented as (mean $\pm \mathrm{SD}$ ) or [median, interquartile range]. When a study did not state uNGAL and/or uKIM-1 concentrations, but presented them in a figure instead, these data were estimated and denoted using . EX, exercise; nr, not reported; HS, heat stress; $\mathrm{VO}_{2}$, maximal oxygen uptake; $\mathrm{MD}$, muscle damaging; $\mathrm{CON}$, control; $\Delta$, change; EU, euhydrated; HYP, hypohydrated; uNGAL, urinary neutrophil gelatinase-associated lipocalin; uKIM-1, urinary kidney injury molecule-1; AKI, acute kidney injury.

\section{Repeated Bouts of Exercise}

An important caveat when interpreting the effect of exercise training/competition on long-term renal function is that exercise training has been suggested to preserve renal function through the improvement of cardiometabol- ic risk factors [10, 33]. To our knowledge, only one study has examined the effect of bouts of exercise on consecutive days on UNGAL and uKIM-1. Bongers et al. [18] found that uncorrected and osmolality-corrected post-exercise uNGAL and uKIM- 1 concentrations did not accumulate after 3 days of prolonged walking. However, the effect of 


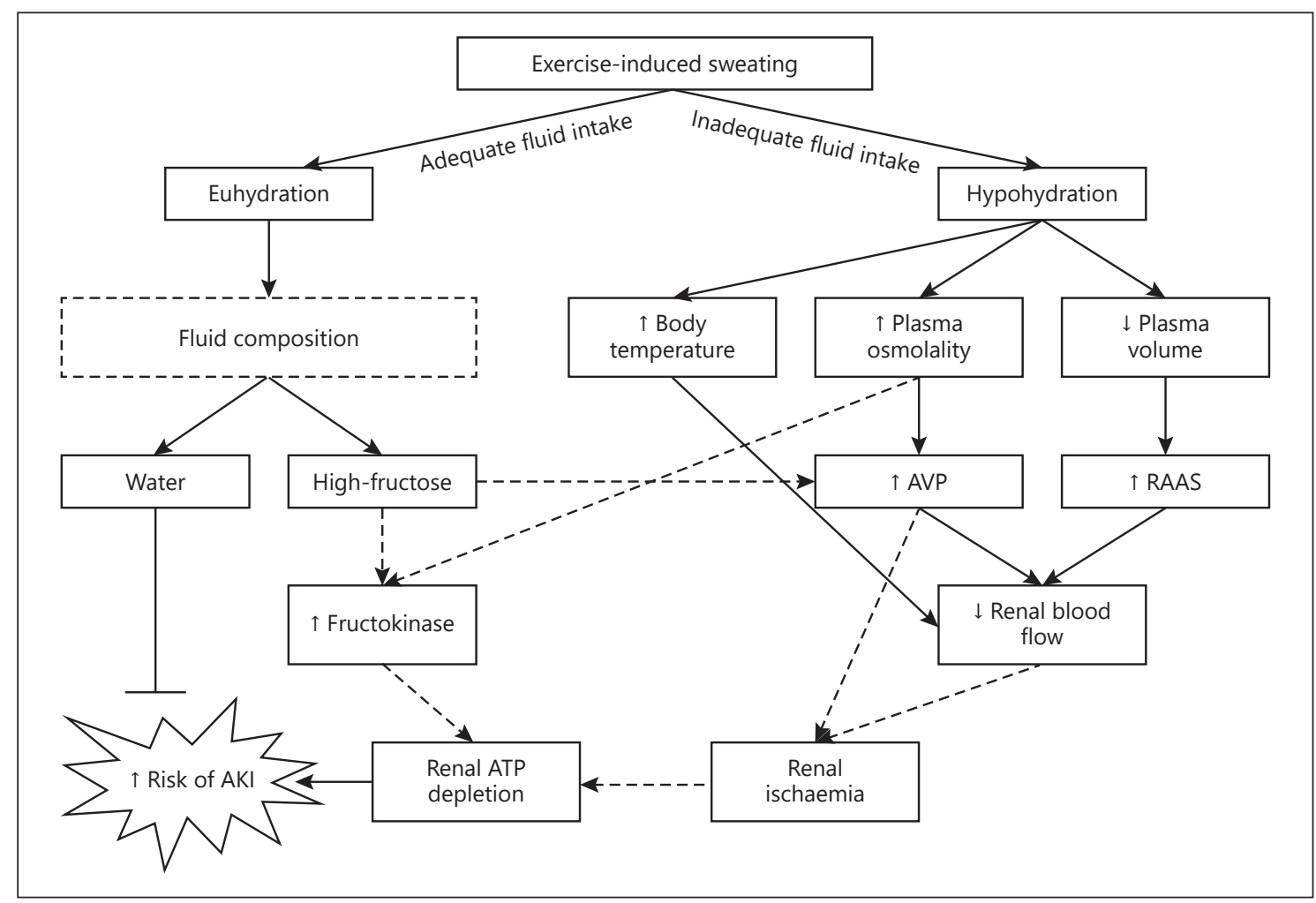

Fig. 2. Schematic representing how fluid intake may influence the risk of exercise-associated AKI. Solid arrows refer to established pathways, dashed arrows refer to potential/conditional pathways and the arrow from water to risk of AKI refers to potential inhibition. Temp, temperature; AVP, arginine vasopressin; RAAS, renin-angiotensin-aldosterone system; ATP, adenosine triphosphate; AKI, acute kidney injury.

repeated bouts of more strenuous exercise, which have been shown to cause higher elevations in novel AKI biomarkers, remains unknown. Serum $\mathrm{Cr}$ has been measured after various stages of multistage ultramarathons, but similarly, no accumulation was found [4]. In addition, individuals that developed exercise-associated AKI from an ultramarathon did not experience greater post-exercise renal dysfunction at a following ultramarathon [1]. In this study, pre-exercise blood samples were not taken, and thus pre-exercise serum $\mathrm{Cr}$ concentrations were approximated [1]. In this instance, it would have been beneficial to have measured pre-exercise serum Crvalues, as $\mathrm{Cr}$ is of more use when it is in steady state. This was done by Pryor et al. [34], who showed that 6 days of heat acclimation did not affect baseline serum Cr. A potential explanation for these findings may be that changes in novel AKI biomarkers, such as UNGAL, may precede changes in serum $\mathrm{Cr}$ [35]. Therefore, further research is required to determine the effect of repeated bouts of strenuous exercise on novel AKI biomarkers in athletic populations.

Effects of Exercise on Acute Kidney Injury Biomarkers

\section{The Potential Role of Fluid Intake in Exercise- Associated AKI}

During exercise, the increase in metabolic heat production results in sweating, and as endurance athletes rarely match their sweat losses with fluid intake, hypohydration is commonly present towards the end of endurance events [6]. Not only can hypohydration reduce RBF but it also has the potential to exacerbate exercise-associated AKI via other mechanisms. As sweat losses are hypotonic compared to plasma, failure to replace them with fluid intake typically causes a decrease in plasma volume and a rise in plasma osmolality that draws water out of the intracellular fluid compartment via osmosis $[26,27]$. This results in the release of arginine vasopressin, which acts on the kidney to increase water reabsorption $[26,27]$. While this process is important for water conservation, it may result in increased renal oxygen consumption [28], which could exacerbate exercise-induced renal ischaemia and subsequent kidney injury. It was recently document- 
ed that marathon runners with AKI had higher post-exercise copeptin (a surrogate marker of vasopressin) concentrations than those without [21]. Plasma hypertonicity may also result in the conversion of glucose to sorbitol in the proximal tubule [36]. Sorbitol can then be converted to fructose, which can be metabolized by fructokinase, potentially resulting in renal ATP deletion and subsequent AKI [36].

While it has been suggested that the most common cause of exercise-associated AKI is muscle damage [1], this may require coexisting hypohydration in order to cause AKI [25]. In a study where large rises in markers of muscle damage were caused by maximal eccentric contractions, but hydration status was controlled for with fluid ingestion, there was no incidence of AKI [25]. A potential explanation for this is that both muscle damage and hypohydration may act in concert to increase kidney injury via synergistic reductions in plasma volume and RAAS activation [23]. Furthermore, while Junglee et al. [15] demonstrated that muscle damage increased uNGAL, it is important to note that this was in the presence of hypohydration.

To our knowledge, only two studies have used a crossover design to investigate the effect of hypohydration during exercise/simulated physical work on novel AKI biomarkers. Butts [20] manipulated hydration status (via fluid ingestion or restriction) before and during exercise in the heat that was preceded by muscle damaging exercise. They showed no rise in uNGAL from pre- to postexercise in either trial, and while uNGAL concentrations were higher in the hypohydrated trial at these time points, correction for urine osmolality removed this effect [20]. Conversely, Chapman et al. [32] showed, through a panel of novel AKI biomarkers, that maintaining euhydration during simulated physical work in the heat appeared to reduce injury to the proximal tubules. These results may explain the lack of effect of hydration status on osmolality-corrected uNGAL in Butts [20], as a rise in uNGAL is thought to indicate injury mainly to the distal nephron [14].

Whilst maintaining euhydration may be beneficial, it is possible that not all drinks produce the same effect. Compared to water, ingestion of a high-fructose soft drink during and after simulated physical work in the heat caused a large increase in AKI incidence and a small increase in overnight uNGAL [31]. This effect may have been mediated through activation of the vasopressin and fructokinase pathways $[31,36]$, and thus the disparity between the large rise in AKI incidence and small increase in overnight uNGAL may be because fructose ingestion is more likely to lead to the injury of the proximal tubules [36]. If uKIM-1 had been measured in this study, we speculate that there would have been a large difference between trials. Therefore, although research is scarce, it is possible that fluid intake (volume and composition) might represent a potential modifiable factor to influence AKI biomarkers/risk (see Fig. 2 for potential effects).

\section{Conclusion}

The increase in serum $\mathrm{Cr}$ following prolonged endurance events, such as marathons and ultramarathons, appears to be accompanied by an increase in novel biomarkers of AKI. This suggests renal tubular injury, rather than just muscle damage and/or a temporary reduction in RBF. The large rise in AKI biomarkers often seen following marathons and ultramarathons is likely due to the long duration and relatively high exercise intensity, resulting in an increase in sympathetic tone, muscle damage, core body temperature and level of hypohydration. While it is challenging to isolate the role of these factors, due to their mechanistic interactions, it has been shown that maintaining euhydration with water ingestion during simulated physical work in the heat attenuated rises in biomarkers of AKI [32]. However, ingesting a highfructose soft drink during simulated physical work in the heat has been shown to exacerbate markers of AKI. Unfortunately, whether these findings regarding fluid intake apply to athletes undertaking strenuous exercise in a temperate environment remains unknown. The long-term effects of exercise-associated increases in AKI biomarkers and the effect of interventions that may attenuate these rises, such as maintaining euhydration with water ingestion, are also not well understood and require further research.

\section{Conflict of Interest Statement}

L.A. Juett received travel expenses and registration fees from Danone Research to attend the 2019 Hydration for Health Scientific Conference. L.J. James has previously received funding for his research from PepsiCo., Inc. and has previously performed consultancy work for Lucozade Ribena Suntory. These previous funding/consultancy activities are in no way linked to the present article and have always been paid directly to L.J. James's employer, not to L.J. James. The authors have no other conflicts of interest to declare.
Juett/James/Mears 


\section{References}

1 Hoffman MD, Weiss RH. Does acute kidney injury from an ultramarathon increase the risk for greater subsequent injury? Clin J Sport Med. 2016;26(5):417-22.

2 Kao WF, Hou SK, Chiu YH, Chou SL, Kuo FC, Wang SH, et al. Effects of $100-\mathrm{km}$ ultramarathon on acute kidney injury. Clin J Sport Med. 2015;25(1):49-54.

3 Mansour SG, Verma G, Pata RW, Martin TG, Perazella MA, Parikh CR. Kidney injury and repair biomarkers in marathon runners. Am J Kidney Dis. 2017;70(2):252-61.

4 Lipman GS, Krabak BJ, Waite BL, Logan SB, Menon A, Chan GK. A prospective cohort study of acute kidney injury in multi-stage ultramarathon runners: the biochemistry in endurance runner study (BIERS). Res Sports Med. 2014;22(2):185-92.

5 Poussel M, Touzé C, Allado E, Frimat L, Hily $\mathrm{O}$, Thilly $\mathrm{N}$, et al. Ultramarathon and renal function: does exercise-induced acute kidney injury really exist in common conditions? Front Sports Act Living. 2020 Jan 21;1:71.

6 Cheuvront SN, Haymes EM. Thermoregulation and marathon running. Sport Med. 2001; 31(10):743-62.

7 Coca SG, Singanamala S, Parikh CR. Chronic kidney disease after acute kidney injury: a systematic review and meta-analysis. Kidney Int. 2012;81(5):442-8.

8 Weiner DE, McClean MD, Kaufman JS, Brooks DR. The central american epidemic of CKD. Clin J Am Soc Nephrol. 2013;8(3):50411.

9 Go AS, Chertow GM, Fan D, McCulloch CE, Hsu CY. Chronic kidney disease and the risks of death, cardiovascular events, and hospitalization. N Engl J Med. 2004;351(13):1296305.

10 Schlader ZJ, Hostler D, Parker MD, Pryor RR, Lohr JW, Johnson BD, et al. The potential for renal injury elicited by physical work in the heat. Nutrients. 2019;11(9):2087.

11 Hodgson L, Walter E, Venn R, Galloway R, Pitsiladis Y, Sardat F, et al. Acute kidney injury associated with endurance events: is it a cause for concern? A systematic review. BMJ Open Sport Exerc Med. 2017;3(1):e000093.

12 Poortmans JR. Exercise and renal function. Sports Med. 1984 Mar-Apr;1(2):125-53.

13 Kashani K, Cheungpasitporn W, Ronco C. Biomarkers of acute kidney injury: the pathway from discovery to clinical adoption. Clin Chem Lab Med. 2017;55(8):1074-89.
14 Helanova K, Spinar J, Parenica J. Diagnostic and prognostic utility of neutrophil gelatinase-associated lipocalin (NGAL) in patients with cardiovascular diseases: review. Kidney Blood Press Res. 2014;39(6):623-9.

15 Junglee NA, Di Felice U, Dolci A, Fortes MB, Jibani MM, Lemmey AB, et al. Exercising in a hot environment with muscle damage: effects on acute kidney injury biomarkers and kidney function. Am J Physiol Renal Physiol. 2013; 305(6):F813-20.

16 Lippi G, Sanchis-Gomar F, Salvagno GL, Aloe R, Schena F, Guidi GC. Variation of serum and urinary neutrophil gelatinase associated lipocalin (NGAL) after strenuous physical exercise. Clin Chem Lab Med. 2012;50(9): $1585-9$.

17 Junglee NA, Lemmey AB, Burton M, Searell C, Jones D, Lawley JS, et al. Does proteinuriainducing physical activity increase biomarkers of acute kidney injury? Kidney Blood Press Res. 2012;36(1):278-89.

18 Bongers CCWG, Alsady M, Nijenhuis T, Hartman YAW, Eijsvogels TMH, Deen PMT, et al. Impact of acute versus repetitive moderate intensity endurance exercise on kidney injury markers. Physiol Rep. 2017;5(24):1-11.

19 Bongers CCWG, Alsady M, Nijenhuis T, Tulp ADM, Eijsvogels TMH, Deen PMT, et al. Impact of acute versus prolonged exercise and dehydration on kidney function and injury. Physiol Rep. 2018;6(11):e13734-11.

20 Butts CL. Dehydration, muscle damage, and exercise in the heat: impacts on renal stress, thermoregulation, and muscular damage recovery. Fayetteville: University of Arkansas; 2018.

21 Mansour SG, Martin TG, Obeid W, Pata RW Myrick KM, Kukova L, et al. The role of volume regulation and thermoregulation in AKI during marathon running. Clin J Am Soc Nephrol. 2019;14(9):1297-305.

22 Radigan LR, Robinson S. Effects of environmental heat stress and exercise on renal blood flow and filtration rate. J Appl Physiol. 1949; 2(4):185-91.

23 Petejova N, Martinek A. Acute kidney injury due to rhabdomyolysis and renal replacement therapy: a critical review. Crit Care. 2014; 18(3):224-8.

24 Basile DP, Anderson MD, Sutton TA. Pathophysiology of acute kidney injury. Compr Physiol. 2012;2(2):1303-53.

25 Clarkson PM, Kearns AK, Rouzier P, Rubin $\mathrm{R}$, Thompson PD. Serum creatine kinase levels and renal function measures in exertional muscle damage. Med Sci Sports Exerc. 2006; 38(4):623-7.
26 Cheuvront SN, Kenefick RW. Dehydration: physiology, assessment, and performance effects. Compr Physiol. 2014;4(1):257-85.

27 James LJ, Funnell MP, James RM, Mears SA. Does hypohydration really impair endurance performance? Methodological considerations for interpreting hydration research. Sports Med. 2019;49(Suppl 2):103-14.

28 Bragadottir G, Redfors B, Nygren A, Sellgren J, Ricksten SE. Low-dose vasopressin increases glomerular filtration rate, but impairs renal oxygenation in post-cardiac surgery patients. Acta Anaesthesiol Scand. 2009;53(8):1052-9.

29 Funnell MP, Mears SA, Bergin-Taylor K, James LJ. Blinded and unblinded hypohydration similarly impair cycling time trial performance in the heat in trained cyclists. J Appl Physiol. 2019;126(4):870-9.

30 McCullough PA, Chinnaiyan KM, Gallagher MJ, Colar JM, Geddes T, Gold JM, et al. Changes in renal markers and acute kidney injury after marathon running. Nephrology. 2011;16(2):194-9.

31 Chapman CL, Johnson BD, Sackett JR, Parker MD, Schlader ZJ. Soft drink consumption during and following exercise in the heat elevates biomarkers of acute kidney injury. Am J Physiol Regul Integr Comp Physiol. 2019; 316(3):R189-98

32 Chapman CL, Johnson BD, Vargas NT, Hostler D, Parker MD, Schlader ZJ. Hyperthermia and dehydration during physical work in the heat both contribute to the risk of acute kidney injury. J Appl Physiol. 2020 Apr 1;128(4): $715-28$.

33 Stump CS. Physical activity in the prevention of chronic kidney disease. Cardiorenal Med. 2011;1(3):164-73.

34 Pryor RR, Pryor JL, Vandermark LW, Adams EL, Brodeur RM, Schlader ZJ, et al. Acute kidney injury biomarker responses to short-term heat acclimation. Int $\mathrm{J}$ Environ Res Public Health. 2020;17(4):1-13.

35 Laws RL, Brooks DR, Amador JJ, Weiner DE, Kaufman JS, Ramírez-Rubio O, et al. Biomarkers of kidney injury among Nicaraguan sugarcane workers. Am J Kidney Dis. 2016; 67(2):209-17.

36 Jimenez CAR, Ishimoto T, Lanaspa MA, Rivard CJ, Nakagawa T, Ejaz AA, et al. Fructokinase activity mediates dehydration-induced renal injury. Kidney Int. 2014;86(2):294-302. 